\title{
Coupled Hilfer fractional differential systems with random effects
}

\author{
Saïd Abbas ${ }^{1}$, Mouffak Benchohra ${ }^{2}$ and Yong Zhou ${ }^{3,4^{*}}$ (D)
}

\section{"Correspondence:}

yzhou@xtu.edu.cn

${ }^{3}$ Faculty of Information Technology,

Macau University of Science and

Technology, Macau, P.R. China

${ }^{4}$ Faculty of Mathematics and

Computational Science, Xiangtan

University, Xiangtan, P.R. China

Full list of author information is

available at the end of the article

\begin{abstract}
This paper deals with some existence results for two classes of coupled systems of Hilfer and Hilfer-Hadamard random fractional differential equations. The main tool used to carry out our results is Itoh's random fixed point theorem.

MSC: 26A33; 45D05; 45G05; 45M10

Keywords: Functional differential equation; Left-sided mixed Riemann-Liouville integral of fractional order; Left-sided mixed Hadamard integral of fractional order; Hilfer fractional derivative; Hilfer-Hadamard fractional derivative; Coupled system; Random solution; Fixed point
\end{abstract}

\section{Introduction}

Fractional differential equations have recently been applied in various areas of engineering, mathematics, physics and bio-engineering, and other applied sciences [13, 23]. For some fundamental results in the theory of fractional calculus and fractional differential equations we refer the reader to the monographs of Abbas et al. [5, 6], Samko et al. [22], and Kilbas et al. [18], and a series of papers [2-4, 7, 15, 26, 27, 29-33] and the references cited therein. Coupled systems of Hadamard type sequential fractional differential equations were considered in $[8,9]$. Recently, considerable attention has been given to the existence of solutions of initial and boundary value problems for fractional differential equations with Hilfer fractional derivative; see [1, 11-13, 16, 24, 28].

The nature of a dynamic system in engineering or natural sciences depends on the accuracy of the information we have concerning the parameters that describe that system. If the knowledge about a dynamic system is precise then a deterministic dynamical system arises. Unfortunately in most cases the available data for the description and evaluation of parameters of a dynamic system are inaccurate, imprecise or confusing. In other words, evaluation of parameters of a dynamical system is not without uncertainties. When our knowledge about the parameters of a dynamic system are of statistical nature, that is, the information is probabilistic, the common approach in mathematical modeling of such systems is the use of random differential equations or stochastic differential equations. Random differential equations, as natural extensions of deterministic ones, arise in many applications and have been investigated by many mathematicians. We refer the reader to Refs. $[10,19,25]$.

(c) The Author(s) 2018. This article is distributed under the terms of the Creative Commons Attribution 4.0 International License (http://creativecommons.org/licenses/by/4.0/), which permits unrestricted use, distribution, and reproduction in any medium, provided you give appropriate credit to the original author(s) and the source, provide a link to the Creative Commons license, and indicate if changes were made. 
In this paper we discuss the existence of solutions for the following coupled random Hilfer fractional differential system:

$$
\left\{\begin{array}{l}
\left(\left(D_{0}^{\alpha_{1}, \beta_{1}} u_{1}\right)(t, w),\left(D_{0}^{\alpha_{2}, \beta_{2}} u_{2}\right)(t, w)\right) \\
\quad=\left(f_{1}\left(t, u_{1}(t, w), u_{2}(t, w), w\right), f_{2}\left(t, u_{1}(t, w), u_{2}(t, w), w\right)\right) ; \quad t \in I, \quad w \in \Omega \\
\left.\left(\left(I_{0}^{1-\gamma_{1}} u_{1}\right)(t, w),\left(I_{0}^{1-\gamma_{2}} u_{2}\right)(t, w)\right)\right|_{t=0}=\left(\phi_{1}(w), \phi_{2}(w)\right),
\end{array}\right.
$$

where $I:=[0, T], T>0, \alpha_{i} \in(0,1), \beta_{i} \in[0,1], \gamma_{i}=\alpha_{i}+\beta_{i}-\alpha_{i} \beta_{i} ; i=1,2 ;(\Omega, \mathcal{A})$ is a measurable space, $\phi_{i}: \Omega \rightarrow \mathbb{R}$ is a measurable function, $f_{i}: I \times \mathbb{R} \times \mathbb{R} \times \Omega \rightarrow \mathbb{R}$ is a given function, $I_{0}^{1-\gamma_{i}}$ is the left-sided mixed Riemann-Liouville integral of order $1-\gamma_{i}$, and $D_{0}^{\alpha_{i}, \beta_{i}}$ is the Hilfer fractional derivative of order $\alpha_{i}$ and type $\beta_{i}$.

Next, we consider the following coupled system of random Hilfer-Hadamard fractional differential equations:

$$
\left\{\begin{array}{l}
\left.\left({ }^{H} D_{1}^{\alpha_{1}, \beta_{1}} u_{1}\right)(t, w),\left({ }^{H} D_{1}^{\alpha_{2}, \beta_{2}} u_{2}\right)(t, w)\right) \\
\quad=\left(g_{1}\left(t, u_{1}(t, w), u_{2}(t, w), w\right), g_{2}\left(t, u_{1}(t, w), u_{2}(t, w), w\right)\right) ; \quad t \in[1, T], \quad w \in \Omega, \\
\left.\left(\left({ }^{H} I_{1}^{1-\gamma_{1}} u_{1}\right)(t, w),\left({ }^{H} I_{1}^{1-\gamma_{2}} u_{2}\right)(t, w)\right)\right|_{t=1}=\left(\psi_{1}(w), \psi_{2}(w)\right),
\end{array}\right.
$$

where $T>1, \alpha_{i} \in(0,1), \beta_{i} \in[0,1], \gamma_{i}=\alpha_{i}+\beta_{i}-\alpha_{i} \beta_{i}, \psi_{i}: \Omega \rightarrow \mathbb{R} ; i=1,2$ is a measurable function, $g_{i}:[1, T] \times \mathbb{R} \times \mathbb{R} \times \Omega \rightarrow \mathbb{R}$ is a given function, ${ }^{H} I_{1}^{1-\gamma_{i}}$ is the left-sided mixed Hadamard integral of order $1-\gamma_{i}$, and ${ }^{H} D_{1}^{\alpha_{i}, \beta_{i}}$ is the Hilfer-Hadamard fractional derivative of order $\alpha_{i}$ and type $\beta_{i}$.

The present paper initiates the study of coupled systems for Hilfer fractional differential equations with random effects.

\section{Preliminaries}

Let $C$ be the Banach space of all continuous functions $v$ from $I$ into $\mathbb{R}$ with the supremum (uniform) norm

$$
\|v\|_{\infty}:=\sup _{t \in I}|v(t)|
$$

As usual, $A C(I)$ denotes the space of absolutely continuous functions from $I$ into $\mathbb{R}$. We denote by $A C^{1}(I)$ the space defined by

$$
A C^{1}(I):=\left\{w: I \rightarrow \mathbb{R}: \frac{d}{d t} w(t) \in A C(I)\right\} .
$$

By $L^{1}(I)$, we denote the space of Lebesgue-integrable functions $v: I \rightarrow \mathbb{R}$ with the norm

$$
\|v\|_{1}=\int_{0}^{T}|v(t)| d t
$$

Let $L^{\infty}(I)$ be the Banach space of measurable functions $u: I \rightarrow \mathbb{R}$ which are essentially bounded, equipped with the norm

$$
\|u\|_{L^{\infty}}=\inf \{c>0:|u(t)| \leq c \text {, a.e. } t \in I\} .
$$


By $C_{\gamma}(I)$ and $C_{\gamma}^{1}(I)$, we denote the weighted spaces of continuous functions defined by

$$
C_{\gamma}(I)=\left\{w:(0, T] \rightarrow \mathbb{R}: t^{1-\gamma} w(t) \in C\right\},
$$

with the norm

$$
\|w\|_{C_{\gamma}}:=\sup _{t \in I}\left|t^{1-\gamma} w(t)\right|
$$

and with

$$
C_{\gamma}^{1}(I)=\left\{w \in C: \frac{d w}{d t} \in C_{\gamma}\right\}
$$

with the norm

$$
\|w\|_{C_{\gamma}^{1}}:=\|w\|_{\infty}+\|w\|_{C_{\gamma}} .
$$

Also, by $\mathcal{C}:=C_{\gamma_{1}} \times C_{\gamma_{2}}$ we denote the product weighted space with the norm

$$
\|(u, v)\|_{\mathcal{C}}=\|u\|_{C_{\gamma_{1}}}+\|v\|_{C_{\gamma_{2}}} .
$$

Definition 2.1 A function $T: \Omega \times \mathbb{R} \rightarrow \mathbb{R}$ is called jointly measurable if $T(\cdot, u)$ is measurable for all $u \in \mathbb{R}$ and $T(w, \cdot)$ is continuous for all $w \in \Omega$.

Definition 2.2 A function $f: I \times \mathbb{R} \times \Omega \rightarrow \mathbb{R}$ is called random Carathéodory if the following conditions are satisfied:

(i) The map $(t, w) \rightarrow f(t, u, w)$ is jointly measurable for all $u \in \mathbb{R}$, and

(ii) the map $u \rightarrow f(t, u, w)$ is continuous for all $t \in I$ and $w \in \Omega$.

Let $E$ be a Banach space and $T: \Omega \times E \rightarrow E$ be a mapping. Then $T$ is called a random operator if $T(w, u)$ is measurable in $w$ for all $u \in E$ and it expressed as $T(w) u=T(w, u)$. In this case we also say that $T(w)$ is a random operator on $E$. A random operator $T(w)$ on $E$ is called continuous (resp. compact, totally bounded and completely continuous) if $T(w, u)$ is continuous (resp. compact, totally bounded and completely continuous) in $u$ for all $w \in \Omega$. The details of completely continuous random operators in Banach spaces and their properties appear in Itoh [14].

Definition 2.3 Let $\mathcal{P}(Y)$ be the family of all nonempty subsets of $Y$ and $C$ be a mapping from $\omega$ into $\mathcal{P}(Y)$. A mapping $T:\{(w, y): w \in \Omega, y \in C(w)\} \rightarrow Y$ is called random operator with stochastic domain $C$ if $C$ is measurable (i.e., for all closed $A \subset Y,\{w \in \Omega, C(w) \cap A \neq$ $\emptyset\}$ is measurable) and for all open $D \subset Y$ and all $y \in Y,\{w \in \Omega: y \in C(w), T(w, y) \in D\}$ is measurable. $T$ will be called continuous if every $T(w)$ is continuous. For a random operator $T$, a mapping $y: \Omega \rightarrow Y$ is called random (stochastic) fixed point of $T$ if for $P$-almost all $w \in \Omega, y(w) \in C(w)$ and $T(w) y(w)=y(w)$ and for all open $D \subset Y,\{w \in \Omega: y(w) \in D\}$ is measurable.

Now, we give some results and properties of fractional calculus. 
Definition 2.4 ([5, 18, 22]) The left-sided mixed Riemann-Liouville integral of order $r>0$ of a function $w \in L^{1}(I)$ is defined by

$$
\left(I_{0}^{r} w\right)(t)=\frac{1}{\Gamma(r)} \int_{0}^{t}(t-s)^{r-1} w(s) d s ; \quad \text { for a.e. } t \in I,
$$

where $\Gamma(\cdot)$ is the (Euler's) Gamma function defined by

$$
\Gamma(\xi)=\int_{0}^{\infty} t^{\xi-1} e^{-t} d t ; \quad \xi>0 .
$$

Notice that, for all $r, r_{1}, r_{2}>0$ and each $w \in C$, we have $I_{0}^{r} w \in C$, and

$$
\left(I_{0}^{r_{1}} I_{0}^{r_{2}} w\right)(t)=\left(I_{0}^{r_{1}+r_{2}} w\right)(t) ; \quad \text { for a.e. } t \in I
$$

Definition $2.5([5,18,22])$ The Riemann-Liouville fractional derivative of order $r \in(0,1]$ of a function $w \in L^{1}(I)$ is defined by

$$
\begin{aligned}
\left(D_{0}^{r} w\right)(t) & =\left(\frac{d}{d t} I_{0}^{1-r} w\right)(t) \\
& =\frac{1}{\Gamma(1-r)} \frac{d}{d t} \int_{0}^{t}(t-s)^{-r} w(s) d s ; \quad \text { for a.e. } t \in I .
\end{aligned}
$$

Let $r \in(0,1], \gamma \in[0,1)$ and $w \in C_{1-\gamma}(I)$. Then the following expression leads to the leftinverse operator.

$$
\left(D_{0}^{r} I_{0}^{r} w\right)(t)=w(t) ; \quad \text { for all } t \in(0, T]
$$

Moreover, if $I_{0}^{1-r} w \in C_{1-\gamma}^{1}(I)$, then the following composition is proved in [22]:

$$
\left(I_{0}^{r} D_{0}^{r} w\right)(t)=w(t)-\frac{\left(I_{0}^{1-r} w\right)\left(0^{+}\right)}{\Gamma(r)} t^{r-1} ; \quad \text { for all } t \in(0, T] .
$$

Definition $2.6([5,18,22])$ The Caputo fractional derivative of order $r \in(0,1]$ of a function $w \in L^{1}(I)$ is defined by

$$
\begin{aligned}
\left({ }^{c} D_{0}^{r} w\right)(t) & =\left(I_{0}^{1-r} \frac{d}{d t} w\right)(t) \\
& =\frac{1}{\Gamma(1-r)} \int_{0}^{t}(t-s)^{-r} \frac{d}{d s} w(s) d s ; \quad \text { for a.e. } t \in I .
\end{aligned}
$$

In [13], Hilfer studied applications of a generalized fractional operator having the Riemann-Liouville and the Caputo derivatives as specific cases (see also [16, 24]).

Definition 2.7 (Hilfer derivative) Let $\alpha \in(0,1), \beta \in[0,1], w \in L^{1}(I), I_{0}^{(1-\alpha)(1-\beta)} \in A C^{1}(I)$. The Hilfer fractional derivative of order $\alpha$ and type $\beta$ of $w$ is defined as

$$
\left(D_{0}^{\alpha, \beta} w\right)(t)=\left(I_{0}^{\beta(1-\alpha)} \frac{d}{d t} I_{0}^{(1-\alpha)(1-\beta)} w\right)(t) ; \quad \text { for a.e. } t \in I .
$$


Properties Let $\alpha \in(0,1), \beta \in[0,1], \gamma=\alpha+\beta-\alpha \beta$, and $w \in L^{1}(I)$.

1. The operator $\left(D_{0}^{\alpha, \beta} w\right)(t)$ can be written as

$$
\left(D_{0}^{\alpha, \beta} w\right)(t)=\left(I_{0}^{\beta(1-\alpha)} \frac{d}{d t} I_{0}^{1-\gamma} w\right)(t)=\left(I_{0}^{\beta(1-\alpha)} D_{0}^{\gamma} w\right)(t) ; \quad \text { for a.e. } t \in I .
$$

Moreover, the parameter $\gamma$ satisfies

$$
\gamma \in(0,1], \quad \gamma \geq \alpha, \quad \gamma>\beta, \quad 1-\gamma<1-\beta(1-\alpha) .
$$

2. The generalization (3) for $\beta=0$, coincides with the Riemann-Liouville derivative and for $\beta=1$ with the Caputo derivative.

$$
D_{0}^{\alpha, 0}=D_{0}^{\alpha} \quad \text { and } \quad D_{0}^{\alpha, 1}={ }^{c} D_{0}^{\alpha} .
$$

3. If $D_{0}^{\beta(1-\alpha)} w$ exists and is in $L^{1}(I)$, then

$$
\left(D_{0}^{\alpha, \beta} I_{0}^{\alpha} w\right)(t)=\left(I_{0}^{\beta(1-\alpha)} D_{0}^{\beta(1-\alpha)} w\right)(t) ; \quad \text { for a.e. } t \in I .
$$

Furthermore, if $w \in C_{\gamma}(I)$ and $I_{0}^{1-\beta(1-\alpha)} w \in C_{\gamma}^{1}(I)$, then

$$
\left(D_{0}^{\alpha, \beta} I_{0}^{\alpha} w\right)(t)=w(t) ; \quad \text { for a.e. } t \in I .
$$

4. If $D_{0}^{\gamma} w$ exists and is in $L^{1}(I)$, then

$$
\left(I_{0}^{\alpha} D_{0}^{\alpha, \beta} w\right)(t)=\left(I_{0}^{\gamma} D_{0}^{\gamma} w\right)(t)=w(t)-\frac{I_{0}^{1-\gamma}\left(0^{+}\right)}{\Gamma(\gamma)} t^{\gamma-1} ; \quad \text { for a.e. } t \in I
$$

Corollary 2.8 Let $h \in C_{\gamma}(I)$. Then the Cauchy problem

$$
\left\{\begin{array}{l}
\left(D_{0}^{\alpha, \beta} u\right)(t)=h(t) ; \quad t \in I, \\
\left.\left(I_{0}^{1-\gamma} u\right)(t)\right|_{t=0}=\phi
\end{array}\right.
$$

has a unique solution given by

$$
u(t)=\frac{\phi}{\Gamma(\gamma)} t^{\gamma-1}+\left(I_{0}^{\alpha} h\right)(t)
$$

From the above corollary, we conclude with the following lemma.

Lemma 2.9 Let $f_{i}: I \times \mathbb{R} \times \mathbb{R} \times \Omega \rightarrow \mathbb{R} ; i=1,2$ be such that $f\left(\cdot, u_{1}(\cdot, w), u_{2}(\cdot, w), w\right) \in C_{\gamma_{i}}$ for all $w \in \Omega$, and any $u_{i}(w) \in C_{\gamma_{i}}$. Then the coupled system (1) is equivalent to the problem of solutions of the following system of fractional integral equations:

$$
u_{i}(t, w)=\frac{\phi_{i}(w)}{\Gamma\left(\gamma_{i}\right)} t^{\gamma_{i}-1}+\left(I_{0}^{\alpha_{i}} f_{i}\left(\cdot, u_{1}(\cdot, w), u_{2}(\cdot, w), w\right)\right)(t) ; \quad w \in \Omega, i=1,2 .
$$

We need the following Itoh random fixed point theorem; see [14]. 
Theorem 2.10 Let $X$ be a nonempty, closed convex bounded subset of the separable Banach space $E$ and let $N: \Omega \times X \rightarrow X$ be a compact and continuous random operator. Then the random equation $N(w) u=u$ has a random solution.

\section{Coupled systems of Hilfer fractional random differential equations}

In this section, we are concerned with the existence of solutions for the system (1). Let us start by defining what we mean by a random solution of the system (1).

Definition 3.1 By a random solution of the problem (1) we mean a coupled measurable function $\left(u_{1}, u_{2}\right): \Omega \rightarrow C_{\gamma_{1}} \times C_{\gamma_{2}}$ that satisfies the conditions $\left(I_{0}^{1-\gamma_{i}} u_{i}\right)\left(0^{+}, w\right)=\phi_{i}(w) ; i=$ 1,2 , and the equations $\left(D_{0}^{\alpha_{i}, \beta_{i}} u_{i}\right)(t, w)=f_{i}\left(t, u_{1}(t, w), u_{2}(t, w), w\right) ; i=1,2$ on $I \times \Omega$.

The following hypotheses will be used in the sequel.

$\left(\mathrm{H}_{1}\right)$ The functions $f_{i} ; i=1,2$ are random Carathéodory on $I \times \mathbb{R} \times \mathbb{R} \times \Omega$,

$\left(\mathrm{H}_{2}\right)$ there exist measurable and bounded functions $p_{i}, q_{i}: \Omega \rightarrow L^{\infty}(I,[0, \infty)) ; i=1,2$, such that

$$
\left|f_{i}\left(t, u_{1}, u_{2}, w\right)\right| \leq \frac{p_{i}(t, w)\left|u_{1}\right|+q_{i}(t, w)\left|u_{2}\right|}{1+\left|u_{1}\right|+\left|u_{2}\right|}
$$

for a.e. $t \in I$, and each $u_{i} \in \mathbb{R}, w \in \Omega$.

Now, we shall prove the following theorem concerning the existence of random solutions of the system (1).

Theorem 3.2 Assume that the hypotheses $\left(\mathrm{H}_{1}\right)$ and $\left(\mathrm{H}_{2}\right)$ hold. Then the system (1) has at least one random solution defined on $I \times \Omega$.

Proof Define the following operators $N_{i}: \Omega \times C_{\gamma_{i}} \rightarrow C_{\gamma_{i}} ; i=1,2$ :

$$
\left(N_{i}(w) u_{i}\right)(t)=\frac{\phi_{i}(w)}{\Gamma\left(\gamma_{i}\right)} t^{\gamma_{i}-1}+\int_{0}^{t}(t-s)^{\alpha_{i}-1} \frac{f_{i}\left(s, u_{1}(s, w), u_{2}(s, w), w\right)}{\Gamma\left(\alpha_{i}\right)} d s,
$$

and consider the continuous operator $N: \Omega \times \mathcal{C} \rightarrow \mathcal{C}$ defined by

$$
N(w)\left(u_{1}, u_{2}\right)=\left(N_{1}(w) u_{1}, N_{2}(w) u_{2}\right)
$$

Set

$$
p_{i}^{*}=\sup _{w \in \Omega}\left\|p_{i}(w)\right\|_{L^{\infty}}, \quad q_{i}^{*}=\sup _{w \in \Omega}\left\|q_{i}(w)\right\|_{L^{\infty}}, \quad \text { and } \quad \phi_{i}^{*}=\sup _{w \in \Omega}\left|\phi_{i}(w)\right| ; \quad i=1,2 .
$$

For each $i=1,2$, the map $\phi_{i}$ is measurable for all $w \in \Omega$. Again, as the indefinite integral is continuous on $I$, then $N_{i}(w)$ defines a mapping $N_{i}: \Omega \times C_{\gamma_{i}} \rightarrow C_{\gamma_{i}}$. Thus $\left(u_{1}, u_{2}\right)$ is a random solution for the system (1) if and only if $\left(u_{1}, u_{2}\right)=N(w)\left(u_{1}, u_{2}\right)$.

Next, for any $u_{i} \in C_{\gamma_{i}} ; i=1,2$, and each $t \in I$ and $w \in \omega$, we have

$$
\begin{aligned}
\left|t^{1-\gamma_{i}}\left(N_{i}(w) u_{i}\right)(t)\right| & \leq \frac{\left|\phi_{i}(w)\right|}{\Gamma\left(\gamma_{i}\right)}+\frac{t^{1-\gamma_{I}}}{\Gamma\left(\alpha_{i}\right)} \int_{0}^{t}(t-s)^{\alpha_{i}-1}\left|f_{i}\left(s, u_{1}(s, w), u_{2}(s, w), w\right)\right| d s \\
& \leq \frac{\left|\phi_{i}(w)\right|}{\Gamma\left(\gamma_{i}\right)}+\frac{t^{1-\gamma_{i}}}{\Gamma\left(\alpha_{i}\right)} \int_{0}^{t}(t-s)^{\alpha_{i}-1}\left(p_{i}(s, w)+q_{i}(s, w)\right) d s
\end{aligned}
$$




$$
\begin{aligned}
& \leq \frac{\phi_{I}^{*}}{\Gamma\left(\gamma_{i}\right)}+\frac{\left(p_{i}^{*}+q_{i}^{*}\right) T^{1-\gamma_{i}}}{\Gamma\left(\alpha_{i}\right)} \int_{0}^{t}(t-s)^{\alpha_{i}-1} d s \\
& \leq \frac{\phi_{i}^{*}}{\Gamma\left(\gamma_{i}\right)}+\frac{\left(p_{i}^{*}+q_{i}^{*}\right) T^{1-\gamma_{i}+\alpha_{i}}}{\Gamma\left(1+\alpha_{i}\right)} .
\end{aligned}
$$

Thus

$$
\left\|N(w)\left(u_{1}, u_{2}\right)\right\|_{\mathcal{C}} \leq \sum_{i=1}^{2} \frac{\phi_{i}^{*}}{\Gamma\left(\gamma_{i}\right)}+\frac{\left(p_{i}^{*}+q_{i}^{*}\right) T^{1-\gamma_{i}+\alpha_{i}}}{\Gamma\left(1+\alpha_{i}\right)}:=R
$$

This proves that $N(w)$ transforms the ball

$$
B_{R}:=B(0, R)=\left\{\left(u_{1}, u_{2}\right) \in \mathcal{C}:\left\|\left(u_{1}, u_{2}\right)\right\|_{\mathcal{C}} \leq R\right\}
$$

into itself. We shall show that the operator $N: \Omega \times B_{R} \rightarrow B_{R}$ satisfies all the assumptions of Theorem 2.10. The proof will be given in several steps.

Step $1 . N(w)$ is a random operator on $\Omega \times B_{R}$ into $B_{R}$.

Since for each $i=1,2, f_{i}\left(t, u_{1}, u_{2}, w\right)$ is random Carathéodory, the map $w \rightarrow f_{i}\left(t, u_{1}, u_{2}, w\right)$ is measurable in view of Definition 2.1. Similarly, the product $(t-s)^{\alpha_{i}-1} f_{i}\left(s, u_{1}(s, w)\right.$, $\left.u_{2}(s, w), w\right)$ of a continuous and a measurable function is again measurable. Further, the integral is a limit of a finite sum of measurable functions, therefore, the map

$$
w \mapsto \frac{\phi_{i}(w)}{\Gamma\left(\gamma_{i}\right)} t^{\gamma_{i}-1}+\int_{0}^{t} \frac{(t-s)^{\alpha_{i}-1}}{\Gamma\left(\alpha_{i}\right)} f_{i}\left(s, u_{1}(s, w), u_{2}(s, w), w\right) d s,
$$

is measurable. As a result, $N(w)$ is a random operator on $\Omega \times B_{R}$ into $B_{R}$.

Step $2 . N(w)$ is continuous.

Let $\left\{\left(u_{1 n}, u_{2 n}\right)\right\}_{n \in \mathbb{N}}$ be a sequence such that $\left(u_{1 n}, u_{2 n}\right) \rightarrow\left(u_{1}, u_{2}\right)$ in $B_{R}$. Then, for each $i=1,2, t \in I$, and $w \in \Omega$, we have

$$
\begin{aligned}
& \left|t^{1-\gamma}\left(N_{i}(w) u_{i n}\right)(t)-t^{1-\gamma_{i}}\left(N_{i}(w) u_{i}\right)(t)\right| \\
& \quad \leq \frac{t^{1-\gamma_{i}}}{\Gamma\left(\alpha_{i}\right)} \int_{0}^{t}(t-s)^{\alpha_{i}-1}\left|f_{i}\left(s, u_{1 n}(s, w), u_{2 n}(s, w), w\right)-f\left(s, u_{1}(s, w), u_{2}(s, w), w\right)\right| d s .
\end{aligned}
$$

Since $\left(u_{1 n}, u_{2 n}\right) \rightarrow\left(u_{1}, u_{2}\right)$ as $n \rightarrow \infty$ and $f_{i}$ is random Carathéodory, then, by the Lebesgue dominated convergence theorem, Eq. (7) implies

$$
\left\|N(w)\left(u_{1 n}, u_{2 n}\right)-N(w)\left(u_{1}, u_{2}\right)\right\|_{\mathcal{C}} \rightarrow 0 \quad \text { as } n \rightarrow \infty .
$$

Step 3. $N(w) B_{R}$ is uniformly bounded.

This is clear since $N(w) B_{R} \subset B_{R}$ and $B_{R}$ is bounded.

Step 4. $N(w) B_{R}$ is equicontinuous.

Let $t_{1}, t_{2} \in I, t_{1}<t_{2}$ and let $\left(u_{1}, u_{2}\right) \in B_{R}$. Then, for each $i=1,2$, and $w \in \Omega$, we have

$$
\begin{aligned}
& \left|t_{2}^{1-\gamma_{i}}\left(N_{i}(w) u_{i}\right)\left(t_{2}\right)-t_{1}^{1-\gamma_{i}}\left(N_{i}(w) u_{i}\right)\left(t_{1}\right)\right| \\
& \quad \leq \mid t_{2}^{1-\gamma_{i}} \int_{0}^{t_{2}}\left(t_{2}-s\right)^{\alpha_{i}-1} \frac{f_{i}\left(s, u_{1}(s, w), u_{2}(s, w), w\right)}{\Gamma\left(\alpha_{i}\right)} d s
\end{aligned}
$$




$$
\begin{aligned}
& -t_{1}^{1-\gamma_{i}} \int_{0}^{t_{1}}\left(t_{1}-s\right)^{\alpha_{i}-1} \frac{f_{i}\left(s, u_{1}(s, w), u_{2}(s, w), w\right)}{\Gamma\left(\alpha_{i}\right)} d s \mid \\
\leq & t_{2}^{1-\gamma_{i}} \int_{t_{1}}^{t_{2}}\left(t_{2}-s\right)^{\alpha_{i}-1} \frac{\left|f_{i}\left(s, u_{1}(s, w), u_{2}(s, w), w\right)\right|}{\Gamma\left(\alpha_{i}\right)} d s \\
& +\int_{0}^{t_{1}}\left|t_{2}^{1-\gamma_{i}}\left(t_{2}-s\right)^{\alpha_{i}-1}-t_{1}^{1-\gamma_{i}}\left(t_{1}-s\right)^{\alpha_{i}-1}\right| \frac{\left|f_{i}\left(s, u_{1}(s, w), u_{2}(s, w), w\right)\right|}{\Gamma\left(\alpha_{i}\right)} d s \\
\leq & t_{2}^{1-\gamma_{i}} \int_{t_{1}}^{t_{2}}\left(t_{2}-s\right)^{\alpha_{i}-1} \frac{p_{i}(s, w)+q_{i}(s, w)}{\Gamma\left(\alpha_{i}\right)} d s \\
& +\int_{0}^{t_{1}}\left|t_{2}^{1-\gamma_{i}}\left(t_{2}-s\right)^{\alpha_{i}-1}-t_{1}^{1-\gamma_{i}}\left(t_{1}-s\right)^{\alpha_{i}-1}\right| \frac{p_{i}(s, w)+q_{i}(s, w)}{\Gamma\left(\alpha_{i}\right)} d s .
\end{aligned}
$$

Thus, we get

$$
\begin{aligned}
& \left|t_{2}^{1-\gamma_{i}}\left(N_{i}(w) u_{i}\right)\left(t_{2}\right)-t_{1}^{1-\gamma_{i}}\left(N_{i}(w) u_{i}\right)\left(t_{1}\right)\right| \\
& \quad \leq \frac{\left(p_{i}^{*}+q_{i}^{*}\right) T^{1-\gamma_{i}+\alpha_{i}}}{\Gamma\left(1+\alpha_{i}\right)}\left(t_{2}-t_{1}\right)^{\alpha_{i}}+\frac{p_{i}^{*}+q_{i}^{*}}{\Gamma\left(\alpha_{i}\right)} \int_{0}^{t_{1}}\left|t_{2}^{1-\gamma_{i}}\left(t_{2}-s\right)^{\alpha_{i}-1}-t_{1}^{1-\gamma_{i}}\left(t_{1}-s\right)^{\alpha_{i}-1}\right| d s .
\end{aligned}
$$

As $t_{1} \longrightarrow t_{2}$, the right-hand side of the above inequality tends to zero.

As a consequence of steps 1 to 4 together with the Arzelá-Ascoli theorem, we can conclude that $N: \Omega \times B_{R} \rightarrow B_{R}$ is continuous and compact. Theorem 2.10 implies that the operator equation $N(w)\left(u_{1}, u_{2}\right)=\left(u_{1}, u_{2}\right)$ has a random solution. This shows that the random system (1) has a random solution.

\section{Hilfer-Hadamard fractional random differential equations}

Now, we are concerned with some existence results for the coupled system (2).

Set $C:=C([1, T])$. Denote the weighted space of continuous functions defined by

$$
C_{\gamma, \ln }([1, T])=\left\{w(t):(\ln t)^{1-\gamma} w(t) \in C\right\}
$$

with the norm

$$
\|w\|_{C_{\gamma, \mathrm{ln}}}:=\sup _{t \in[1, T]}\left|(\ln t)^{1-r} w(t)\right| .
$$

Let us recall some definitions and properties of Hadamard fractional integration and differentiation. We refer to [18] for a more detailed analysis.

Definition 4.1 (Hadamard fractional integral [18]) The Hadamard fractional integral of order $q>0$ for a function $g \in L^{1}([1, T])$, is defined as

$$
\left({ }^{H} I_{1}^{q} g\right)(x)=\frac{1}{\Gamma(q)} \int_{1}^{x}\left(\ln \frac{x}{s}\right)^{q-1} \frac{g(s)}{s} d s
$$

provided the integral exists.

Example 4.2 Let $0<q<1$. Then

$$
{ }^{H} I_{1}^{q} \ln t=\frac{1}{\Gamma(2+q)}(\ln t)^{1+q}, \quad \text { for a.e. } t \in[0, e] .
$$


Set

$$
\delta=x \frac{d}{d x}, \quad q>0, \quad n=[q]+1,
$$

and

$$
A C_{\delta}^{n}:=\left\{u:[1, T] \rightarrow \mathbb{R}: \delta^{n-1}[u(x)] \in A C(I)\right\} .
$$

Analogous to the Riemann-Liouville fractional calculus, the Hadamard fractional derivative is defined in terms of the Hadamard fractional integral in the following way.

Definition 4.3 (Hadamard fractional derivative [18]) The Hadamard fractional derivative of order $q>0$ applied to the function $w \in A C_{\delta}^{n}$ is defined as

$$
\left({ }^{H} D_{1}^{q} w\right)(x)=\delta^{n}\left({ }^{H} I_{1}^{n-q} w\right)(x) .
$$

In particular, if $q \in(0,1]$, then

$$
\left({ }^{H} D_{1}^{q} w\right)(x)=\delta\left({ }^{H} I_{1}^{1-q} w\right)(x)
$$

Example 4.4 Let $0<q<1$. Then

$$
{ }^{H} D_{1}^{q} \ln t=\frac{1}{\Gamma(2-q)}(\ln t)^{1-q}, \quad \text { for a.e. } t \in[0, e] .
$$

It has been proved (see e.g. Kilbas [17, Theorem 4.8]) that in the space $L^{1}(I, \mathbb{R})$, the Hadamard fractional derivative is the left-inverse operator to the Hadamard fractional integral, i.e.

$$
\left({ }^{H} D_{1}^{q}\right)\left({ }^{H} I_{1}^{q} w\right)(x)=w(x) .
$$

From Theorem 2.3 of [18], we have

$$
\left({ }^{H} I_{1}^{q}\right)\left({ }^{H} D_{1}^{q} w\right)(x)=w(x)-\frac{\left({ }^{H} I_{1}^{1-q} w\right)(1)}{\Gamma(q)}(\ln x)^{q-1} .
$$

Analogous to the Hadamard fractional calculus, the Caputo-Hadamard fractional derivative is defined in the following way.

Definition 4.5 (Caputo-Hadamard fractional derivative) The Caputo-Hadamard fractional derivative of order $q>0$ applied to the function $w \in A C_{\delta}^{n}$ is defined as

$$
\left({ }^{H c} D_{1}^{q} w\right)(x)=\left({ }^{H} I_{1}^{n-q} \delta^{n} w\right)(x)
$$

In particular, if $q \in(0,1]$, then

$$
\left({ }^{H c} D_{1}^{q} w\right)(x)=\left({ }^{H} I_{1}^{1-q} \delta w\right)(x) .
$$


From the Hadamard fractional integral, the Hilfer-Hadamard fractional derivative (introduced for the first time in [20]) is defined in the following way.

Definition 4.6 (Hilfer-Hadamard fractional derivative) Let $\alpha \in(0,1), \beta \in[0,1], \gamma=\alpha+$ $\beta-\alpha \beta, w \in L^{1}(I)$, and ${ }^{H} I_{1}^{(1-\alpha)(1-\beta)} w \in A C^{1}(I)$. The Hilfer-Hadamard fractional derivative of order $\alpha$ and type $\beta$ applied to the function $w$ is defined as

$$
\begin{aligned}
\left({ }^{H} D_{1}^{\alpha, \beta} w\right)(t) & =\left({ }^{H} I_{1}^{\beta(1-\alpha)}\left({ }^{H} D_{1}^{\gamma} w\right)\right)(t) \\
& =\left({ }^{H} I_{1}^{\beta(1-\alpha)} \delta\left({ }^{H} I_{1}^{1-\gamma} w\right)\right)(t) ; \quad \text { for a.e. } t \in[1, T] .
\end{aligned}
$$

This new fractional derivative (4.6) may be viewed as interpolating the Hadamard fractional derivative and the Caputo-Hadamard fractional derivative. Indeed for $\beta=0$ this derivative reduces to the Hadamard fractional derivative and when $\beta=1$, we recover the Caputo-Hadamard fractional derivative.

$$
{ }^{H} D_{1}^{\alpha, 0}={ }^{H} D_{1}^{\alpha} \quad \text { and } \quad{ }^{H} D_{1}^{\alpha, 1}={ }^{H c} D_{1}^{\alpha} .
$$

From Theorem 21 in [21], we concluded the following lemma.

Lemma 4.7 Let $g_{i}: I \times \mathbb{R} \times \mathbb{R} \times \Omega \rightarrow \mathbb{R} ; i=1,2$ be such that $g_{i}\left(\cdot, u_{1}(\cdot, w), u_{2}(\cdot, w), w\right) \in$ $C_{\gamma_{i}, \ln }([1, T])$ for any $u_{i}(\cdot, w) \in C_{\gamma_{i}, \ln }([1, T])$. Then problem (2) is equivalent to the following system of fractional integral equations:

$$
u_{i}(t, w)=\frac{\psi_{i}(w)}{\Gamma\left(\gamma_{i}\right)}(\ln t)^{\gamma_{i}-1}+\left({ }^{H} I_{1}^{\alpha_{i}} g_{i}\left(\cdot, u_{1}(\cdot, w), u_{2}(\cdot, w), w\right)\right)(t) ; \quad w \in \Omega ; i=1,2 .
$$

Now we give (without proof) an existence result for the system (2). Let us introduce the following hypotheses.

$\left(\mathrm{H}_{1}^{\prime}\right)$ The functions $g_{i} ; i=1,2$ are random Carathéodory on $[1, T] \times \mathbb{R} \times \mathbb{R} \times \Omega$,

$\left(\mathrm{H}_{2}^{\prime}\right)$ there exist measurable and bounded functions $p_{i}, q_{i}: \Omega \rightarrow L^{\infty}([1, T],[0, \infty))$, such that

$$
\begin{aligned}
& \left|g_{i}\left(t, u_{1}, u_{2}, w\right)\right| \leq \frac{p_{i}(t, w)\left|u_{1}\right|+q_{i}(t, w)\left|u_{2}\right|}{1+\left|u_{1}\right|+\left|u_{2}\right|} \\
& \quad \text { for a.e. } t \in[1, T], \text { and each } u_{i} \in \mathbb{R}, w \in \Omega .
\end{aligned}
$$

Theorem 4.8 Assume that the hypotheses $\left(\mathrm{H}_{1}^{\prime}\right)$ and $\left(\mathrm{H}_{2}^{\prime}\right)$ hold. Then the coupled system (2) has at least one random solution defined on $[1, T] \times \Omega$.

\section{An example}

Let $\Omega=(-\infty, 0)$ be equipped with the usual $\sigma$-algebra consisting of Lebesgue measurable subsets of $(-\infty, 0)$. As an application of our results we consider the following system of random Hilfer fractional differential equations:

$$
\begin{cases}\left(D_{0}^{\frac{1}{2}, \frac{1}{2}} u\right)(t, w)=f(t, u(t, w), v(t, w), w) ; & t \in[0,1], \\ \left(D_{0}^{\frac{1}{2}, \frac{1}{2}} v\right)(t, w)=g(t, u(t, w), v(t, w), w) ; & t \in[0,1], \quad w \in \Omega, \\ \left.\left(I_{0}^{\frac{1}{4}} u\right)(t, w)\right|_{t=0}=\left.\left(I_{0}^{\frac{1}{4}} v\right)(t, w)\right|_{t=0}=\frac{1}{1+w^{2}}, & \end{cases}
$$


where

$$
\begin{aligned}
& \left\{\begin{array}{l}
f(t, u, v, w)=\frac{c t^{\frac{-1}{4}}|u| \sin t}{64(1+\sqrt{t})\left(1+w^{2}+|u|+|v|\right)} ; \quad t \in(0,1], u, v \in \mathbb{R}, w \in \Omega, \\
f(0, u, v, w)=0 ; \quad u, v \in \mathbb{R}, w \in \Omega,
\end{array}\right. \\
& g(t, u, v, w)=\frac{c t^{\frac{1}{4}}|v|}{64\left(1+w^{2}+|u|+|v|\right)} ; \quad t \in[0,1], u, v \in \mathbb{R}, w \in \Omega,
\end{aligned}
$$

and $c=\frac{9 \sqrt{\pi}}{16}$. Clearly, the functions $f$ and $g$ are random Carathéodory.

The hypothesis $\left(\mathrm{H}_{2}\right)$ is satisfied with

$$
\left\{\begin{array}{l}
p_{1}(t, w)=\frac{c t^{\frac{-1}{4}}|\sin t|}{64(1+\sqrt{t})\left(1+w^{2}\right)} ; \quad t \in(0,1], w \in \Omega, \\
p_{1}(0, w)=0 ; \quad w \in \Omega,
\end{array}\right.
$$

and $q_{1}(t, w)=p_{2}(t, w)=0, q_{2}(t, w)=\frac{c t \frac{1}{4}}{64} ; t \in(0,1], w \in \Omega$. Hence, Theorem 3.2 implies that the coupled system (9) has at least one random solution defined on $[0,1] \times \Omega$.

\section{Funding}

The work was supported by the National Natural Science Foundation of China (No. 11671339).

Availability of data and materials

Not applicable.

\section{Competing interests}

The authors declare that they have no competing interests.

\section{Authors' contributions}

Each of the authors, SA, MB and YZ contributed equally to each part of this work. All authors read and approved the final manuscript.

\section{Author details}

'Laboratory of Mathematics, Geometry, Analysis, Control and Applications, Tahar Moulay University of Saïda, Saïda, Algeria. ${ }^{2}$ Laboratory of Mathematics, Djillali Liabes University of Sidi Bel-Abbès, Sidi Bel-Abbès, Algeria. ${ }^{3}$ Faculty of Information Technology, Macau University of Science and Technology, Macau, P.R. China. ${ }^{4}$ Faculty of Mathematics and Computational Science, Xiangtan University, Xiangtan, P.R. China.

\section{Publisher's Note}

Springer Nature remains neutral with regard to jurisdictional claims in published maps and institutional affiliations.

Received: 4 August 2018 Accepted: 2 October 2018 Published online: 11 October 2018

\section{References}

1. Abbas, S., Benchohra, M., Graef, J.: Coupled systems of Hilfer fractional differential inclusions in Banach spaces. Commun. Pure Appl. Anal. 17(6), 2479-2493 (2018)

2. Abbas, S., Benchohra, M., Henderson, J., Lazreg, J.E.: Measure of noncompactness and impulsive Hadamard fractional implicit differential equations in Banach spaces. Math. Eng. Sci. Aerosp. 8, 1-19 (2017)

3. Abbas, S., Benchohra, M., Lagreg, J.-E., Alsaedi, A., Zhou, Y.: Existence and Ulam stability for fractional differential equations of Hilfer-Hadamard type. Adv. Differ. Equ. 2017, 180 (2017)

4. Abbas, S., Benchohra, M., Lazreg, J.E., Zhou, Y.: A survey on Hadamard and Hilfer fractional differential equations: analysis and stability. Chaos Solitons Fractals 102, 47-71 (2017)

5. Abbas, S., Benchohra, M., N'Guérékata, G.M.: Topics in Fractional Differential Equations. Springer, New York (2012)

6. Abbas, S., Benchohra, M., N'Guérékata, G.M.: Advanced Fractional Differential and Integral Equations. Nova Science Publishers, New York (2015)

7. Ahmad, B., Alsaedi, A., Kirane, M.: Nonexistence results for the Cauchy problem of time fractional nonlinear systems of thermoelasticity. Math. Methods Appl. Sci. 40, 4272-4279 (2017)

8. Aljoudi, S., Ahmad, B., Nieto, J.J., Alsaedi, A.: A coupled system of Hadamard type sequential fractional differential equations with coupled strip conditions. Chaos Solitons Fractals 91, 39-46 (2016)

9. Aljoudi, S., Ahmad, B., Nieto, J.J., Alsaedi, A.: On coupled Hadamard type sequential fractional differential equations with variable coefficients and nonlocal integral boundary conditions. Filomat 31(19), 6041-6049 (2017)

10. Bharucha-Reid, A.T.: Random Integral Equations. Academic Press, New York (1972) 
11. Furati, K.M., Kassim, M.D.: Non-existence of global solutions for a differential equation involving Hilfer fractional derivative. Electron. J. Differ. Equ. 2013, 235 (2013)

12. Furati, K.M., Kassim, M.D., Tatar, N.-E.: Existence and uniqueness for a problem involving Hilfer fractional derivative. Comput. Math. Appl. 64, 1616-1626 (2012)

13. Hilfer, R.: Applications of Fractional Calculus in Physics. World Scientific, Singapore (2000)

14. Itoh, S.: Random fixed point theorems with applications to random differential equations in Banach spaces. J. Math. Anal. Appl. 67, 261-273 (1979)

15. Jiao, F., Zhou, Y.: Existence results for fractional boundary value problem via critical point theory. Int. J. Bifurc. Chaos 22(4), $1250086(2012)$

16. Kamocki, R., Obczńnski, C.: On fractional Cauchy-type problems containing Hilfer's derivative. Electron. J. Qual. Theory Differ. Equ. 2016, 50 (2016)

17. Kilbas, A.A.: Hadamard-type fractional calculus. J. Korean Math. Soc. 38, 1191-1204 (2001)

18. Kilbas, A.A., Srivastava, H.M., Trujillo, J.J.: Theory and Applications of Fractional Differential Equations. Elsevier, Amsterdam (2006)

19. Ladde, G.S., Lakshmikantham, V:: Random Differential Inequalities. Academic Press, New York (1980)

20. Qassim, M.D., Furati, K.M., Tatar, N.-E.: On a differential equation involving Hilfer-Hadamard fractional derivative. Abstr. Appl. Anal. 2012, Article ID 391062 (2012)

21. Qassim, M.D., Tatar, N.-E.: Well-posedness and stability for a differential problem with Hilfer-Hadamard fractional derivative. Abstr. Appl. Anal. 2013, Article ID 605029 (2013)

22. Samko, S.G., Kilbas, A.A., Marichev, O.I.: Fractional Integrals and Derivatives: Theory and Applications. Gordon \& Breach, Amsterdam (1987). Engl. Trans. from the Russian

23. Tarasov, V.E.: Fractional Dynamics: Application of Fractional Calculus to Dynamics of Particles, Fields and Media. Springer, Heidelberg; Higher Education Press, Beijing (2010)

24. Tomovski, Ž., Hilfer, R., Srivastava, H.M.: Fractional and operational calculus with generalized fractional derivative operators and Mittag-Leffler type functions. Integral Transforms Spec. Funct. 21, 797-814 (2010)

25. Tsokos, C.P., Padgett, W.J.: Random Integral Equations with Applications to Life Sciences and Engineering. Academic Press, New York (1974)

26. Wang, J.R., Feckan, M., Zhou, Y.: A survey on impulsive fractional differential equations. Fract. Calc. Appl. Anal. 19, 806-831 (2016)

27. Wang, J.R., Feckan, M., Zhou, Y.: Center stable manifold for planar fractional damped equations. Appl. Math. Comput. $296,257-269(2017)$

28. Wang, J.R., Zhang, Y.: Nonlocal initial value problems for differential equations with Hilfer fractional derivative. Appl. Math. Comput. 266, 850-859 (2015)

29. Zhou, Y:: Attractivity for fractional evolution equations with almost sectorial operators. Fract. Calc. Appl. Anal. 21(3), 786-800 (2018)

30. Zhou, Y., Ahmad, B., Alsaedi, A.: Existence of nonoscillatory solutions for fractional neutral differential equations. Appl. Math. Lett. 72, 70-74 (2017)

31. Zhou, Y., Shangerganesh, L., Manimaran, J., Debbouche, A.: A class of time-fractional reaction-diffusion equation with nonlocal boundary condition. Math. Methods Appl. Sci. 41, 2987-2999 (2018)

32. Zhou, Y., Vijayakumar, V., Murugesu, R.: Controllability for fractional evolution inclusions without compactness. Evol. Equ. Control Theory 4, 507-524 (2015)

33. Zhou, Y., Zhang, L.: Existence and multiplicity results of homoclinic solutions for fractional Hamiltonian systems. Comput. Math. Appl. 73, 1325-1345 (2017)

\section{Submit your manuscript to a SpringerOpen ${ }^{\circ}$ journal and benefit from:}

- Convenient online submission

- Rigorous peer review

- Open access: articles freely available online

- High visibility within the field

Retaining the copyright to your article

Submit your next manuscript at $\gg$ springeropen.com 